\title{
Training methods and analysis of races of a top level Paralympic swimming athlete
}

\author{
Luca Puce 1,* , Lucio Marinelli', Emanuela Pierantozzi', Laura Mori', Ilaria Pallecchi², Marco Bonifazi ${ }^{3}$, Marco Bove4, \\ Emerson Franchini5 ${ }^{5,6}$, Carlo Trompetto ${ }^{1}$ \\ 'Department of Neuroscience, Rehabilitation, Ophthalmology, Genetics, Maternal and Child Health University of Genoa, Genoa, Italy \\ ${ }^{2}$ CNR-SPIN, Physics Department University of Genoa, Genoa, Italy \\ ${ }^{3}$ Department of Physiology, University of Siena, Siena, Italy \\ ${ }^{4}$ Department of Experimental Medicine, Section of Human Physiology, University of Genoa, Genoa, Italy \\ ${ }^{5}$ Sport Department, University of Sao Paulo, Sao Paulo, Brazil \\ ${ }^{6}$ Australian Institute of Sport, Canberra, Australia
}

Training methods for Paralympic swimmers must take into account different pathologies, competitions classes, athlete's individual circumstances and peculiar physical adaptation mechanisms, hence general guidelines cannot be found in literature. In this study we present a training program, implemented for the physical preparation of a top level Paralympic swimmer. The athlete under study, affected by infantile cerebral palsy within a clinical picture of a spastic tetraparesis, by the end of 2016 was holder of Italian, European, world and Paralympic titles in the 400-m freestyle competition, $\mathrm{S6}$ class. The training macrocycle was structured in a 3-fold periodization (three mesocycles), in view of the preparation to three international competitions. The 4-month training mesocycles prior to each competition differed substantially in terms of mileage load, intensity and recovery times. The first mesocycle was characterized by a sizeable low-intensity mileage load, the second one was shifted to lower mileage load, carried out at middle-to-high intensi- ty levels, the third one entailed increased effort intensity, counterbalanced by lower mileage load. In all cases, recovery times were balanced to obtain optimized performance through physical adaptation to training stimuli, keeping into account the physiopatological response. Tapering phases were adjusted to maximize performance at competition. As an assessment of the effectiveness of the training method, correspondence between chronometric and technical parameters in the three competitions and the respective mesocycle training programs was found. The results of the present study may support the development of training guidelines for athletes affected by upper motor neuron lesions.

Keywords: Sports for the disabled, Physiopathological adaptation, Cerebral palsy spastic, Physical training human, Lactic acid, Anaerobic threshold

\section{INTRODUCTION}

Paralympic swimming is one of the sporting disciplines managed by the International Paralympic Committee (IPC) and involves a sizeable number of athletes. As many as 4,337 athletes, out of which 593 swimmers, took part in the 2016 Rio de Janeiro Paralympic Games (www.paralympic.org/results/historical), attracting extensive media coverage. Training prescription for Paralympic swimmers must take into account the different health conditions, the IPC classes (measuring the degree of physical or intellectual impairment, according to the International "Functional Classification System" [Gehlsen and Karpuk, 1992]) and the individual circumstances of Paralympic swimmers (Fulton et al., 2010).

In swimming, the training load is the overall amount of work required by an athlete to develop short- and long-term adaptive responses, eventually yielding to performance improvement (Costill et al., 1991; Hellard et al., 2005; Mujika, 1998; Mujika et al., 1996b).

In any training program targeted to a specific competition, it is
${ }^{*}$ Corresponding author: Luca Puce (iD https://orcid.org/0000-0003-0825-2707 University of Genoa, Department of Neuroscience, Rehabilitation, Ophthalmology, Genetics, Maternal and Child Health, L.go P. Daneo, 3-16132 Genoa, Italy Tel: +390103537040, Fax: +3901035338720, E-mail: lucalpuce@gmail.com Received: May 9, 2018 / Accepted: June 16, 2018
This is an Open Access article distributed under the terms of the Creative Commons Attribution Non-Commercial License (http://creativecommons.org/licenses/by-nc/4.0/) which permits unrestricted non-commercial use, distribution, and reproduction in any medium, provided the original work is properly cited. 
crucial that peak physical performance be achieved at a precise moment (i.e., competition). Indeed, a tapering stage prior to the competition is designed in such a way that the athlete recovers from previous heavy training loads through a progressive reduction of the load, without undermining the improvements in physical adaptation (Mujika and Padilla, 2003; Mujika et al., 1996a). This goal is obtained by reducing the mileage load, but not the intensity of the training (Mujika and Padilla, 2003; Neary et al., 2003b; Shepley et al., 1992). The effectiveness of the tapering method depends on a number of factors, namely duration, amount of load reduction, type of load reduction, size of previous load (Kubukeli et al., 2002).

To our knowledge, there are no publications on training methods for Paralympic swimmers, and a case study of a high-level Paralympic swimmer training program can contribute to the understanding of training adaptation and its effects on competitive performance. Thus, in the present study, we present the training program of an elite Italian swimmer preparing and wining World, European and Paralympic competitions.

\section{MATERIALS AND METHODS}

\section{Participant characteristics}

The athlete under study is Francesco Bocciardo. He was born in Genoa (Italy) on March 18th 1994, $70 \mathrm{~kg}$ of body mass, 1.71-m height, $9 \%$ of body fat, affected by infantile cerebral palsy spastic tetraparesis. He belongs to the Nuotatori Genovesi civil sport club and to the Guardia Forestale military institution. His IPC categories in the time period analyzed in this work were S6, SM6, and SB5. In 2014-2015, prior to the time period analyzed in this work, his syndrome underwent a severe worsening, involving decreased strength and coordination in the upper limbs, and accentuation of the typical positive effects of upper motor neuron lesions. Consequently, the IPC class was lowered by one unit.

In 2015, he obtained his first international gold medal at the IPC Swimming World Championships held in Glasgow, in the 400-m freestyle race. In 2016 he took part in the IPC Swimming European Open Championships in Funchal, where he obtained gold and bronze medals. On September the 13th 2016 he was gold medalist at the Paralympic Games in Rio de Janeiro, in the S6 class 400-m freestyle competition.

The athlete signed an informed consent document to authorize the publication of his personal details in the present work.

The present study has been carried out in accordance with The Code of Ethics of the World Medical Association (Declaration of
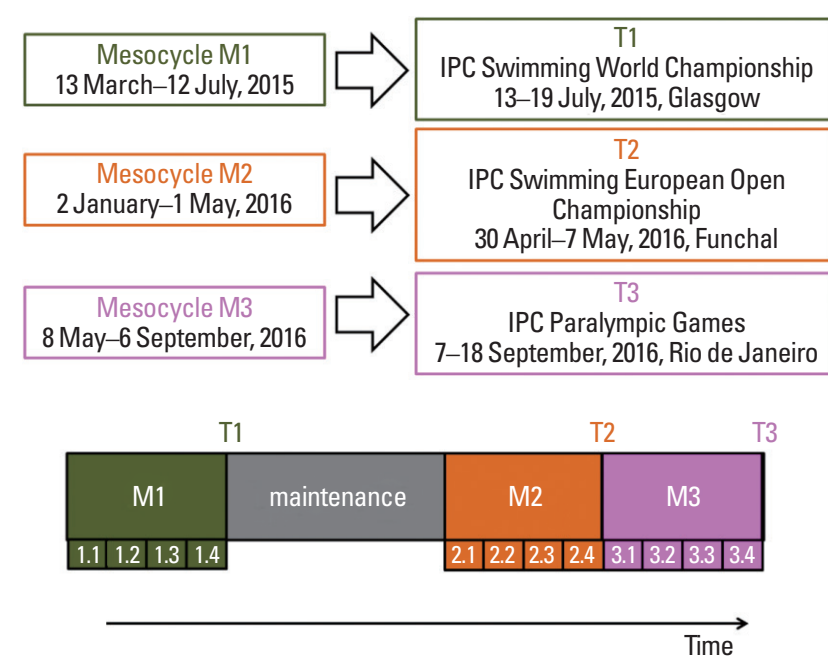

Fig. 1. Upper panel: diagram of competitions and respective training methods, with relevant dates. Lower panel: scaled time axis of the training macrocyle. IPC, International Paralympic Committee.

Helsinki) for experiments involving humans. The project was approved by the local Ethics committee CER-Liguria, date of approval March 29th, 2013, protocol number 46/2012.

\section{Study design}

In this case study, carried out between February 13th 2015 and September 7th 2016, the athlete performed a training macrocycle made up of three successive mesocycles (M1, M2, and M3), each one lasting four months, preparing to three international competitions: M1 for the Glasgow 2105 IPC Swimming World Championships, July 13th-19th, (T1), M2 for the 2016 Funchal IPC Swimming European Open Championships, April 30th-May 7th (T2), M3 for the 2016 Rio de Janeiro Paralympic Games, September 7th-18th (T3). In turn, each mesocycle was subdivided into four 1-month microcycles (Fig. 1). In the 6-month gap between the $\mathrm{T} 1$ competition and the beginning of the $\mathrm{M} 2$ training, the athlete and the training staff arranged a program of low-intensity and low-mileage load maintenance training, to allow for a psycho-physical break. Between the T2 competition and the beginning of the M3 training, only a few days gap occurred. Throughout the four months of each training mesocycle, the athlete took part in several regional and national competition events, which did not alter the planned training, but instead can be considered as racing speed sessions of the training program itself.

\section{Technical details}

In the training sessions, the heart rate was monitored by means 
of a bluetooth chest heart rate monitor (Polar V800 GPS, Polar, Kempele, Finland), which also detects and records distance, speed, number of strokes and rest times. At the end of each training session, data were downloaded, registered and analyzed using the Polar Flow application (Polar, Kempele, Finland). In selected training sessions, the blood lactate concentration [La] was measured by a blood lactate analyzer (Accutrend Plus, Roche Diagnostics $\mathrm{GmbH}$, Mannheim, Germany), by drawing a drop of blood from the earlobe a few seconds after the training test; the muscle hypertonia was assessed in terms of resistance to passive movement of joints and limbs on the basis of the modified Ashworth scale (MAS); the limb pain was assessed by self-perception on the basis of the numeric rating scale (NRS); the body layout and coordination while swimming and clomus were evaluated by visual observation. More precise information on the body layout, with overall as well as detailed view, was obtained by placing adhesive markers on the lower limb joints and on the head, whose position versus time was recorded by four underwater videocameras placed at regular intervals along the pool. Video analysis was carried out with the freeware "The digital coach" (Longomatch "The digital coach," Fluendo, Barcelona, Spain).

As for the analysis of the three races, several studies carried out on able-bodied swimmers demonstrated that the stroke length and the stroke rate (or equivalently stroke count) are suitable parameters to assess the swimming velocity and consequently the race time (Craig and Pendergast, 1979; Craig et al., 1985). Similar results have been obtained on nonexpert swimmers (Pelayo et al., 1997) and swimmers with physical impairments (Pelayo et al., 1999). The stroke index (Costill et al., 1985), defined as the prod- uct of average velocity and stroke length, is considered an indicator of the swimming efficiency. In the present study, the parameters split times, average stroke length, stroke count, stroke rate, and stroke index were monitored throughout the races at even intervals and analyzed.

The measurements of the race parameters were carried out using a videotape footage (software Windows Media Player, Microsoft, Redmond, WA, USA), recorded through three fixed video-cameras recording (GoPro Hero 4, GoPro, San Mateo, CA, USA), placed at either ends of the swimming pool and at the middle point $(25 \mathrm{th} \mathrm{m})$. The split times were acquired using a chronometer (Finis $3 \times 300 \mathrm{M}$ stopwatch, Finis, Livermore, CA, USA). As for the stroke parameters, although it is customary to assess them from the 15 th to the 45 th $\mathrm{m}$ of each $50-\mathrm{m}$ pool length, so as to consider just the free-swimming segments, exempt of dive, turn and apnea stages, for the athlete under study assessing stroke parameters over the whole $50-\mathrm{m}$ pool length gives a more representative and precise evaluation of the swimming performance, because the specific impairment of the athlete affecting mainly lower limbs entails no dive at start, almost no thrust from the pool walls at turns and very limited apnea segments. Hence, the stroke count was measured by counting the number of strokes and the stroke length was calculated as the ratio of swum space $(50 \mathrm{~m})$ to the corresponding number of strokes. For the stroke count, it is assumed that the cycle from the start of one arm pull to the start of the next arm pull made by the same arm (for example from the right-hand entry to the next righthand entry) corresponds to two strokes. Stroke rate and stroke index were calculated from the above parameters.

Table 1. Classification of the training paces, based on guidelines of the FIN and adapted to match the physical characteristics of the Paralympic athlete under examination

\begin{tabular}{|c|c|c|c|c|c|c|c|}
\hline Pace & Regime & $\begin{array}{l}\text { Total time } \\
\text { (min) }\end{array}$ & $\begin{array}{l}\text { Total mileage load } \\
(\mathrm{m})\end{array}$ & $\begin{array}{l}\text { Fractional distances } \\
\text { (m) }\end{array}$ & Rest time between repetitions & $\begin{array}{l}\text { Heart rate } \\
\text { (bpm) }\end{array}$ & $\begin{array}{l}\text { Blood lactate [La] } \\
\text { (mmol/L) }\end{array}$ \\
\hline \multirow[t]{2}{*}{ Aerobic endurance } & A1 & Variable & $2,500-3,500$ & $100-150-200-300-400$ & $10-15-20-25-25 \mathrm{sec}$ & $120-130$ & $\leq 2$ \\
\hline & A2 & $45-60$ & $2,500-3,000$ & $100-150-200-300$ & $10-15-20-25 \mathrm{sec}$ & $<150$ & $\leq 3$ \\
\hline $\begin{array}{l}\text { Aerobic capacity } \\
\text { (anaerobic threshold) }\end{array}$ & B1 & $20-35$ & $1,500-2,500$ & $100-150-200-250$ & $10-10-20-30 \mathrm{sec}$ & $160-170$ & $3-5$ \\
\hline $\begin{array}{l}\text { Aerobic capacity } \\
\text { (maximum stimulus } \\
\text { of aerobic system) }\end{array}$ & B2 & $10-20$ & $800-1,600$ & $\begin{array}{l}200 \text { and } 400 \text { split into } \\
\text { 25-50-100 }\end{array}$ & $\begin{array}{l}\text { 3-5 min between } 200 \text { and } 400 \\
\text { blocks, 3-5-10 sec between } \\
\text { fractions }\end{array}$ & $>180$ & $4-8$ \\
\hline Anaerobic capacity & $\mathrm{C} 1$ & $\leq 8$ & $\leq 600$ & $50-100-150$ & $45-75-120 \mathrm{sec}$ & Maximum & $8-10$ \\
\hline Blood lactate peak & C2 & $3-4$ & $\leq 300$ & $50-75$ & $\begin{array}{l}2-4 \text { min or active recovery in } \\
\text { A1 pace }\end{array}$ & Maximum & Maximum \\
\hline $\begin{array}{l}\text { Anaerobic alactic power: } \\
\text { maximum speed }\end{array}$ & C3 & $2-3$ & $200-300$ & $10-15-20$ & $\begin{array}{l}3 \text { min or active recovery in } \mathrm{A} 1 \\
\text { pace }\end{array}$ & $\begin{array}{l}\text { Below maximum } \\
\text { threshold }\end{array}$ & $\leq 3$ \\
\hline Racing pace & D & 5 & 400 & $25-50-75-100-150$ & Variable & Maximum & $5-8$ \\
\hline
\end{tabular}




\section{RESULTS}

\section{Training method}

\section{Load parameters of the macrocycle}

The training program is described hereafter in terms of load parameters in the macrocycle (Table 1), their effect on the physio- pathologic response of the athlete (Table 2) and their relative distribution throughout the three mesocycles (Fig. 2). Typical examples of training sessions are also presented (Fig. 3).

The load parameters are expressed in terms of: volume, total and split mileage load in training sessions; time, total duration of the training session and density (expressed in terms of duration of

Table 2. Physiopathological response of the athlete in the different training paces

\begin{tabular}{lcccc}
\hline Pace & $\begin{array}{c}\text { Lower limb hypertonia } \\
\text { (MAS) }\end{array}$ & $\begin{array}{c}\text { Lower limb pain } \\
\text { (NRS) }\end{array}$ & Lower limb clonus \\
\hline A1, A2, C3 & 2 & 0 & Absent & Head coordination \\
B1, B2 & 3 & $4-6$ & Possibly present and exhaustible
\end{tabular}

MAS, modified Ashworth scale; NRS, numeric rating scale.

In the body layout sketches, markers placed on the head and on the lower limb joints are indicated by red dots.
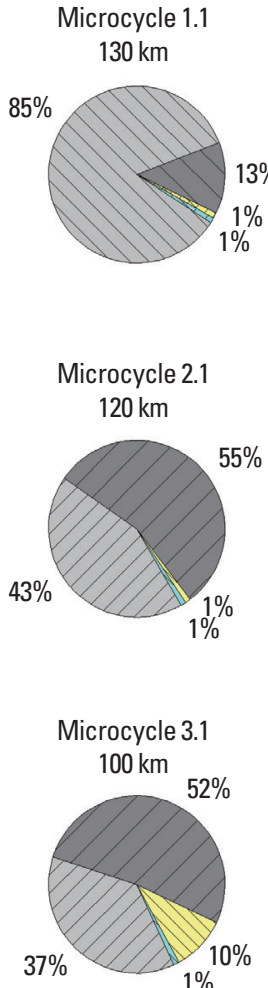

Microcycle 1.2 $170 \mathrm{~km}$
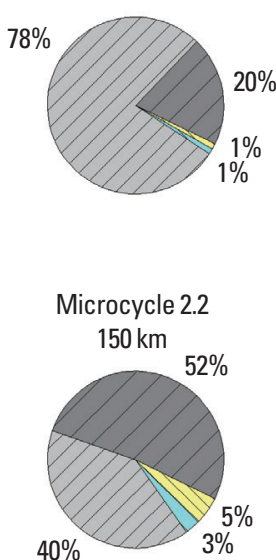

Microcycle 3.2 $130 \mathrm{~km}$

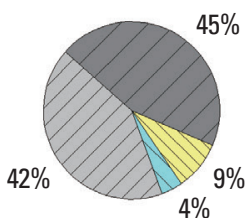

$\mathrm{A} 1, \mathrm{~A} 2$

\section{Mesocycle M1}

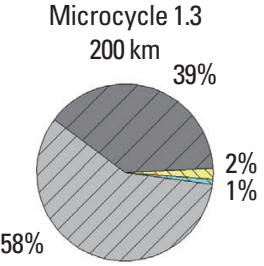

Mesocycle M2
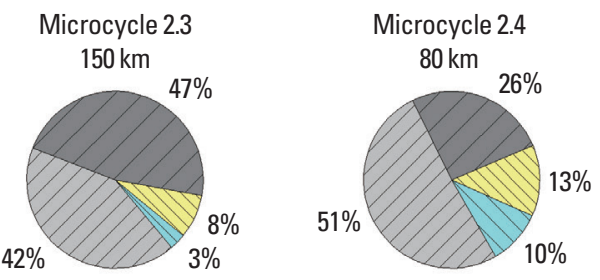

\section{Mesocycle M3}
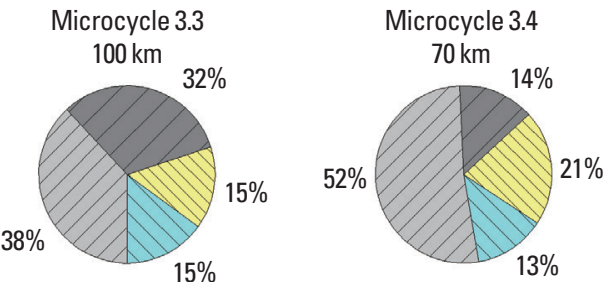

B1, B2

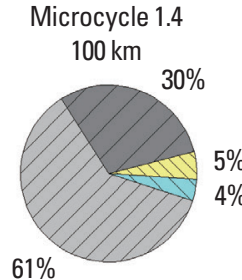

$61 \%$
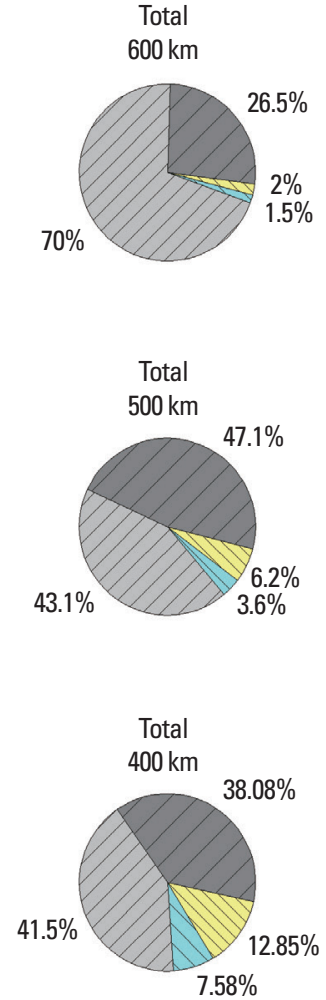

Fig. 2. Pie histograms of swum kilometers in the three mesocycles, subdivided into training paces. 


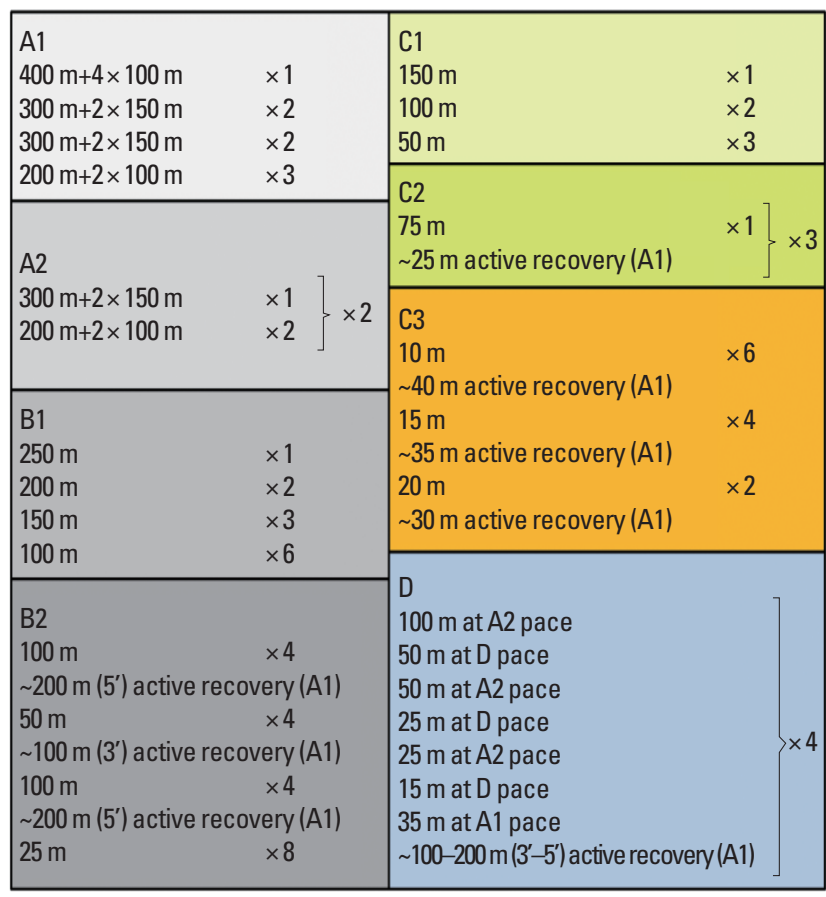

Fig. 3. Examples of typical training sessions.

recovery times between split exercises); intensity, heart rate and [La] at different paces; frequency of the training sessions.

The load parameters are classified into different pace regimes, as reported in Table 1, which was prepared by adapting the guidelines of the Italian Swimming Federation (FIN), so as to comply with the characteristics of the Paralympic athlete under examination. The pace regimes are: aerobic endurance (A: $A 1, A 2)$, aerobic capacity (B: B1, B2), anaerobic system pathways (C: C1, C2, C3), racing pace $(D)$.

The $\mathrm{A} 1$ regime was characterized by slow paces and was used in exercises aimed at improving the swimming technique, as well as in warm-up, cool-down, and recovery stages. In the A2 regime, paces were aimed at improving aerobic endurance. The $[\mathrm{La}]$ and heart rates in $\mathrm{A} 1$ and $\mathrm{A} 2$ paces were $\leq 3 \mathrm{mmol} / \mathrm{L}$ and $120-140$ bpm, respectively. In these regimes, 2 MAS hypertonia, nul NRS, absence of clonus, head coordination under control, and regular body layout were observed. The B1 regime was characterized by middle-to-high levels of physical exertion close to the anaerobic threshold, aimed at improving such threshold, with [La] of 3-5 $\mathrm{mmol} / \mathrm{L}$ and heart rates of $150-160 \mathrm{bpm}$. B1 was the limiting pace that the athlete was able to keep up steadily for 25-30 min. In the $\mathrm{B} 2$ regime the aerobic system was maximally stimulated, with $[\mathrm{La}]$ of $4-8 \mathrm{mmol} / \mathrm{L}$ and heart rates larger than $160 \mathrm{bpm}$. In this regime, the athlete was able to keep up a pace speed which was higher than the B1 speed by $5 \%$, for a typical duration around 7 minutes. The physiopathologic response in the B1 and B2 regimes was characterized by 3 MAS hypertonia, 4-6 NRS, clonus possibly present, yet exhaustible, head coordination under control and regular body layout. In the $\mathrm{C} 1, \mathrm{C} 2$, and $\mathrm{C} 3$ regimes, physical performances of short duration and high-intensity determined maximum or close to maximum heart rates and, for $\mathrm{C} 1$ and $\mathrm{C} 2$, maximum $[\mathrm{La}]$. The $\mathrm{C} 1$ and $\mathrm{C} 2$ regimes were the most critical in triggering the effects of his syndrome, namely muscle hypertonia (4 MAS), likely appearance of inexhaustible clonus, muscle pain (9 NRS), dystonic motion of the head. The body layout was negatively affected by all these effects, specifically lower limbs sank and the ankles were bent at $90^{\circ}$. These effects manifested in the latest stages of the $\mathrm{C} 1$ and $\mathrm{C} 2$ training sessions and increased in intensity up to the end of the session. They typically extinguished after 25-30 minutes of complete rest. A specific study of the correlation between [La] and such effects is unaddressed so far in literature. The $\mathrm{C} 1$ regime is defined as anaerobic capacity, which is the ability to maintain a pace speed with muscles in conditions of acidosis, without negative effects on the technical performance. The $\mathrm{C} 2$ regime is defined as $[\mathrm{La}]$ peak, which is the maximum amount of [La] that the athlete can accumulate. The intensities of training sessions at $\mathrm{C} 2$ pace were higher than those at $\mathrm{C} 1$, hence the mileage loads were nearly halved $(\leq 300 \mathrm{~m})$ and the split distances were reduced (typically $50-75 \mathrm{~m}$ ). As for the $\mathrm{C} 1$ pace, the recovery between repetitions had to be complete, possibly carried out as active recovery by swimming at slow pace. The $\mathrm{C} 3$ pace was aimed at pursuing the improvement of the maximum swimming speed through the improvement of the swimming mechanics and the enhancement of the muscular power. In this case, the maximum split distances were $20 \mathrm{~m}$, swum in times smaller than $10 \mathrm{sec}$ and with complete recovery between repetitions. From the metabolic point of view, $\mathrm{C} 3$ corresponds to the phosphagens system, which was less invasive for the effects of the athlete's health condition as compared to glycolytic paces. The physiopathologic response was analogous to that of the aerobic A1 and $\mathrm{A} 2$ regimes. The $\mathrm{D}$ pace, differently from the other paces, did not correspond to a specific metabolic stimulus, but rather consisted of simulation of racing paces along split distances. The $\mathrm{D}$ pace represents a key and innovative concept, since throughout a single repetition the athlete alternated medium-to-low intensity paces and racing paces, with intervals for complete rest between successive repetitions. We refer to this method as "pace chance training". For example, a typical succession of consecutive paces was A2-DA2-D-A2-D-A1 (Table 1, Fig. 3). Herein, A2 paces were used to 
preactivate the athlete prior to the pace change to $\mathrm{D}$, while $\mathrm{A} 1$ paces were used for active recovery. The peculiar features of this method were: (a) the athlete started each split distance at $\mathrm{D}$ pace from a condition of fatigue, that progressively increased, making it more challenging to sustain the required pace; (b) the athlete could not make full use of his phosphocreatine supply, which would be the case if the exercise was alternated by rest intervals instead of being continuous. The potential target of the $\mathrm{D}$ pace was improving the ability of the athlete to perform different pace changes in the 400-m freestyle competition, without detrimental effects on the performance in the latest stages of the competition.

Fig. 3 presents typical training sessions associated to each of these paces, whereas Fig. 2 describes pie chart histograms representing the mileage load used in the three training mesocycles, subdivided into 1-month microcycles and into pace regimes.

\section{Tapering phase}

In this training program, the tapering phase lasted about 30 days for all the three mesocycles and was visualized in the pie charts of the microcycles 1.4, 2.4, and 3.4 (fourth column of pies in Fig. 2). Significant mileage reductions from $30 \%$ to $50 \%$ were applied in these tapering microcycles. Contextually to the mileage load reduction, the intensity of training at $\mathrm{C}$ and $\mathrm{D}$ paces was increased in all the tapering phases $1.4,2.4$, and 3.4 by $3 \%$ to $8 \%$ with respect to the immediately previous microcycles.

\section{Summary of quantitative differences among mesocycles $M 1, M 2$, and $M 3$}

In the last column of pies in Fig. 2, we present total mileage load swum in the three mesocycles, subdivided into paces. These data are summarized below for easy comparison:

- M1: total mileage load $600 \mathrm{~km}$, of which $70 \%$ for aerobic endurance (A1, A2), 26.5\% for aerobic capacity (B1, B2) and only $3.5 \%$ for anaerobic regimes $(\mathrm{C} 1-3, \mathrm{D})$.

-M2: total mileage load $500 \mathrm{~km}$, of which $43.1 \%$ for aerobic endurance (A1, A2), $47.1 \%$ for aerobic capacity (B1, B2) and $9.8 \%$ for anaerobic regimes $(\mathrm{C} 1-3, \mathrm{D})$.

. M3: total mileage load $400 \mathrm{~km}$, of which $41.5 \%$ for aerobic endurance (A1, A2), 38.1\% for aerobic capacity (B1, B2) and as much as $20.4 \%$ for anaerobic regimes $(C 1-3, D)$, mainly $\mathrm{C} 1$ and $\mathrm{C} 3$.

Hence the three mesocycles differed from one another in terms of total mileage load (M1: 600 km, M2: 500 km, M3: 400 km), relative amount of aerobic endurance paces (M1: 70\%, M2: 43.1\%, M3: 41.5\%), relative amount of aerobic capacity paces
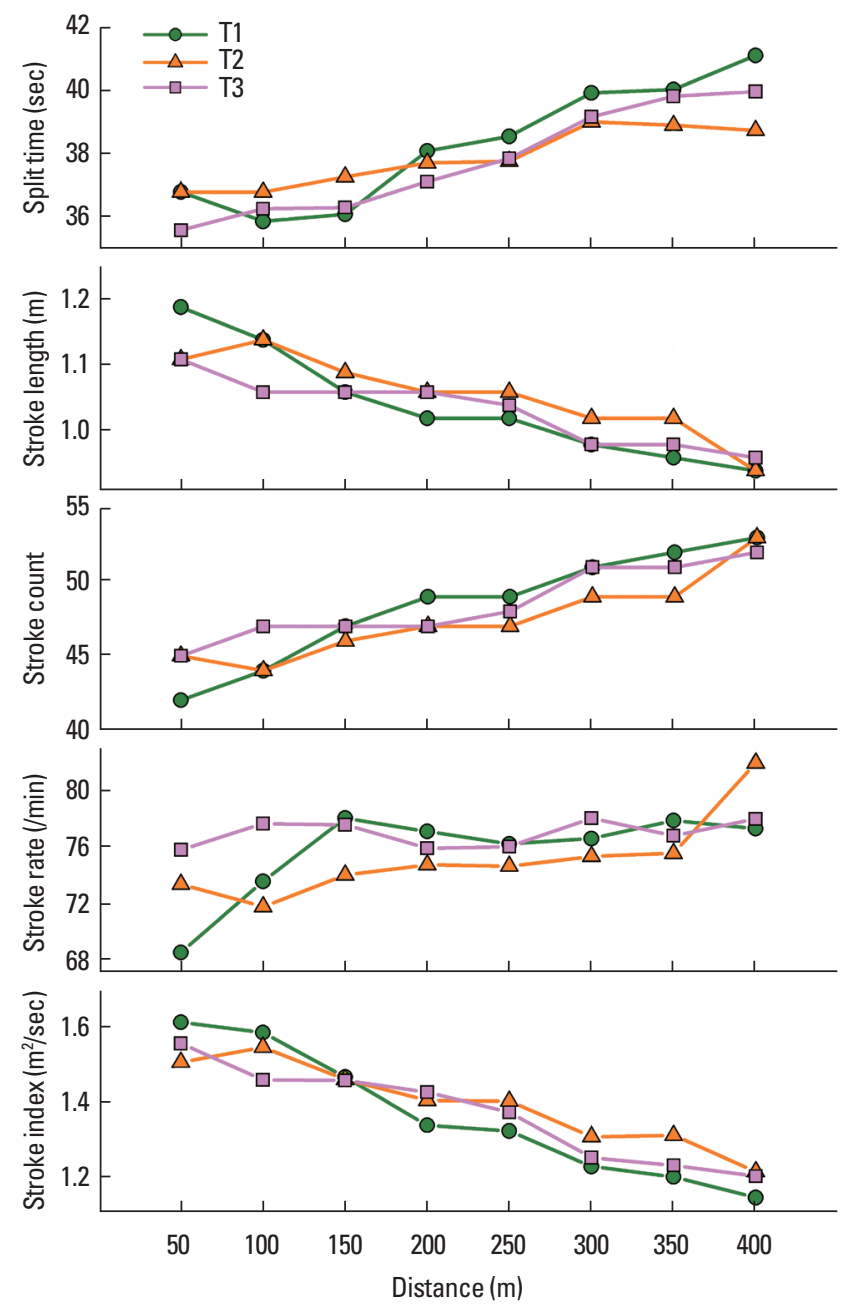

Fig. 4. Split time, average stroke length, stroke count, stroke rate, and stroke index over 50-m lengths recorded in the three competitions T1, T2, and T3 at even 50-m intervals.

(M1: 26.5\%, M2: 47.1\%, M3: 38\%), relative amount of anaerobic paces (M1: 2\%, M2 6.2\%, M3: 12.9\%), relative amount of racing paces (M1: 1.5\%, M2: 3.6\%, M3: 7.6\%).

\section{Chronometric parameters of races $\mathrm{T} 1, \mathrm{~T} 2$, and $\mathrm{T} 3$}

Fig. 4 presents speed, average stroke length, number of strokes, stroke rate and index over $50 \mathrm{~m}$ split distances recorded in the three competitions (T1, T2, and T3). The overall race times and average speeds of the three competitions are summarized below:

$. \mathrm{T} 1: 5 \mathrm{~min} 06.49 \mathrm{sec}$ (speed: $1.31 \mathrm{~m} / \mathrm{sec}$ ).

.T2: $5 \mathrm{~min} 03.02 \mathrm{sec}$ (speed: $1.32 \mathrm{~m} / \mathrm{sec}$ ).

.T3: $5 \mathrm{~min} 02.15 \mathrm{sec}$ (speed: $1.33 \mathrm{~m} / \mathrm{sec}$ ).

The analysis of the split times, stroke length, stroke count, stroke rate, and Costill stoke index are rich in information and 
useful to test the effectiveness of the training methods and of the race strategies of pace change (Fig. 4). Stroke length and stroke count both contribute to the swimming speed and the combination of variables that is right for an athlete depends on several factors. Among these factors, the predominant one was the body layout while swimming, which was modified depending on [La] (compare Tables 1, 2). For $[\mathrm{La}] \sim 10 \mathrm{mmol} / \mathrm{L}$, typical of races, the body layout departed from the streamlined position, so that the athlete experienced a significantly larger drag force and consequently a decrease of the propulsion efficiency. Indeed, it is clearly seen (Fig. 4) that the propulsion effectiveness of the stroke (see the trends of stroke length and stroke index) decreased steadily throughout T1, T2, and T3 competitions, as a consequence of the increased fatigue.

\section{DISCUSSION}

\section{Assessment of the race performances}

From the presented chronometric results, it turns out that the equivalent yearly improvement of the performance time calculated over the three competitions was $1.2 \%$, well above the average $0.5 \%$, resulting from the statistical analysis carried out by Fulton et al. (2009) on 242 top level Paralympic swimmers. Moreover, this $1.2 \%$ improvement of the performance time is also within the range indicated by the same authors for athletes aiming at increasing substantially their medal prospects. Thus, it can be concluded that the training method was effective in improving the athlete's performance.

From the race analysis (Fig. 4), the steadily decreased propulsion effectiveness of the stroke (stroke length and stroke index) throughout the T1, T2, and T3 competitions was much larger than what observed in able-bodied athletes (Schnitzler et al., 2009). In T2, the best stroke length was recorded, with an average value of $0.11 \mathrm{~m}$ and a decrease from the first to the last length as low as $0.08 \mathrm{~m}$. This improvement was due to the fact that the athlete conducted the race with a more steady and gradual development of the pace, thus delaying the $[\mathrm{La}]$ accumulation. In T1 and $\mathrm{T} 3$, the fast split times in the second and first 50-m lengths were responsible for accumulation of $[\mathrm{La}]$ and consequent decrease of average stroke length from the first to the last length by 0.25 $\mathrm{m}$. However, in T3, oppositely to what happened in T1, the athlete managed to keep a fairly good propulsion effectiveness up to the end of the race (Costill stoke index), due to proper training aimed at enduring high $[\mathrm{La}]$ and performing pace changes in the M3 mesocycle (see percentages of $\mathrm{C} 1, \mathrm{C} 2$, and $\mathrm{D}$ paces in $\mathrm{M} 3$ in
Fig. 2, third row).

\section{Comparison of the training method of the athlete under study with methods for able-bodied athletes}

With respect to the load parameters designed for able-bodied athletes by the FIN (available from didactic material for base level, I level and II level coaches, sector technical training), the common aspects are: split distances in training sessions, duration of recovery times, intensity defined by paces. Conversely, the total mileage volume in the training sessions, and consequently also their durations, were different:

.$-50 \%$ mileage volume at $\mathrm{A} 2$ pace

.$-36 \%$ mileage volume at $\mathrm{B} 1$ pace

.$-20 \%$ mileage volume at $\mathrm{B} 2$ pace

- $-40 \%$ mileage volume at $\mathrm{C} 1$ pace

- similar mileage volume at $\mathrm{C} 2$ and $\mathrm{C} 3$ paces

.$-78 \%$ mileage volume at $\mathrm{D}$ pace

In training programs for able-bodied athletes, the stage of maximum load is recommended around four weeks in advance of the competition and the beginning of the tapering phase is recommended between the third-to-last and the second-to-last weeks prior to the competition (Trappe et al., 2001), while for our athlete the phase of maximum load was scheduled between 6 and 5 weeks in advance of the competition, followed by the beginning of the tapering phase at the fourth-to-last weeks.

It has been shown that for able-bodied athletes, the best results are obtained by a mileage load reduction of $31 \%$ in case of no previous overload and by $39 \%$ in case of previous overload, and with a tapering phase duration of 14-28 days, depending on the characteristics of the previous training (Thomas and Busso, 2005). For our athlete the tapering phase was 2 days longer than what is recommended for able-bodied athletes and the mileage reduction was larger than able-bodied values in the first two mesocycles, even larger than $24 \%$, which has been recommended for Paralympic swimmers (Fulton et al., 2010). Indeed, this extra mileage reduction was necessarily applied due to the specificity of the spastic tetraparesis pathology. However, in some qualitative aspects the used tapering method was consistent with that applied to able-bodied athletes. In particular, it was characterized by a larger amount of base endurance training at low-intensity paces $(A)$ and lower amount of base endurance training at high-intensity paces (B). Indeed, training at pace $\mathrm{A}$ was the most suitable to maintain the optimal performance level up to the time of the race, taking advantage of the metabolic adaptation and of the muscle fiber contractile properties generated by the short high-intensity train- 
ings typical of the tapering phases (Mujika et al., 2004; Neary et al., 2003a; Shepley et al., 1992; Trappe et al., 2001).

\section{Physical response to training of an athlete affected by upper motor neuron lesions}

Although the presented year-long macrocycle training had a 3 -fold periodization targeted at three specific competitions, the three 4-month mesocycles were interdependent and contributed to the overall steady improvement of the athlete performance, culminating in the success at the $\mathrm{T} 3$ competition. Indeed, due to the physical adaptation induced by the stimulation of the aerobic metabolism, which occurred throughout the first two training macrocycles (70\% and $43 \%$ of aerobic activity in M1 and M2, respectively), training mesocycles M1 and M2 could have contributed to the physical preparation of the athlete, allowing him to tackle higher intensity trainings in $\mathrm{M} 3$.

In the mesocycle M3, with high anaerobic content, special care was taken regarding recovery times and high-intensity efforts, planning a suitable balance between these two factors. Indeed, the positive effects typical of his pathology turned out to be very sensitive to the stresses induced by the glycolytic metabolism, causing increased spastic hypertone, pain, stiffness and clomus in the leg muscles. Specific studies to get insight into the role of intense and prolonged physical activity in modifying the neuro-physio-pathological mechanisms of the upper motor neuron syndrome and adaptation processes should be carried out. In this work, we observed that the development of the adaptation mechanisms induced by the physical exertion occurs in four stages (Issurin, 2009), which differ in the cases of an able-bodied athlete and a

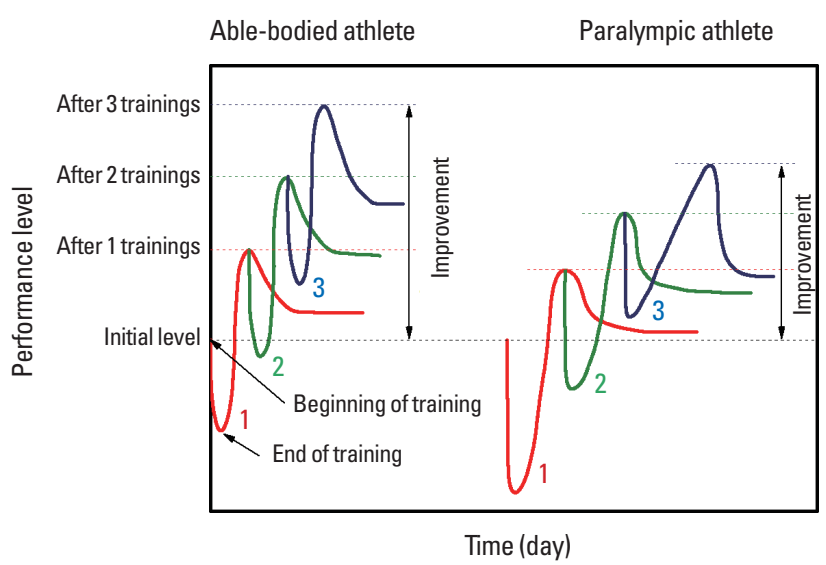

Fig. 5. Sketch of the physical adaptation curves for an able-bodied athlete and a para-athlete affected by spastic tetraparesis, across three successive cycles of training and rest phases. spastic para-athlete (Fig. 5). The first stage is characterized by a decreased athletic efficiency occurring throughout and just after the training session; for equal amounts of physical exertion and duration of workout, the performance decrement is more evident in the para-athlete. In the second stage, at rest, it takes longer to the para-athlete to recover the initial performance level as compared to the able-bodied athlete. In the third stage, the supercompensation mechanism yields an improvement of the performance at rest, with respect to the initial level, yet the edge of improvement in the para-athlete is smaller as compared to the able-bodied athlete. The fourth stage describes an occurrence which was not explored in this study, namely in absence of a new training stimulus the performance level of the para-athlete or able-bodied athlete alike tends to go back to its value prior to the stress induced by the physical training. A key factor for the observed successful achievements was the precise determination of the optimized timing in the second and third stages of physical preparation (Siff, 2004). In particular, in the second stage, whenever the para-athlete was subject to a racing pace training before full recovery from the previous training session, the adaptive response did not keep up with expectations and an abrupt performance drop was observed, causing a physical and psychological overload in the worst cases. In the third stage, the identification of the supercompensation peak was of utmost importance, as this is the optimal physical state for the para-athlete to get a new training stimulus and maximize the cumulative effects of successive training sessions. The curves sketched in Fig. 5 are merely qualitative, as the quantitative details depend on multifold variables such as the physical and psychological states of the para-athlete and external elements such as water temperature and ventilation of the swimming pool environment.

In conclusion, assuming that the excellent competitive achievements are closely related to the effectiveness of the adopted training protocol, guidelines can be extracted for athletes affected by upper motor neuron lesions. In a year-long training program, high mileage loads aiming at improving endurance should be preponderant in the first 4-month mesocycle. In this phase, aerobic regimes should be targeted at improving the swimming technique. In the following 4-month mesocycle, the total mileage load should be decreased by around $15 \%$. In this second phase, the relative fraction of anaerobic regime should be increased, while maintaining at the same time a relatively large fraction of endurance regime. In the last 4-month mesocycle, just prior to the competition event, the mileage load should be further decreased by $20 \%$, planning middle-to-short distance training ses- 
sions, characterized by speeds at racing pace or larger, and longer rest times. Throughout the whole macrocycle, but even more importantly in the last phase, mild aerobic sessions and intervals of passive recovery must be steadily introduced after high-intensity sessions. The subtle adjustment of such recovery and rest times is the key issue to the successful outcome of any training method that must take into account the physical peculiarities of any para-athlete affected by upper motor neuron lesions.

\section{CONFLICT OF INTEREST}

No potential conflict of interest relevant to this article was reported.

\section{REFERENCES}

Costill DL, Kovaleski J, Porter D, Kirwan J, Fielding R, King D. Energy expenditure during front crawl swimming: predicting success in middle-distance events. Int J Sports Med 1985;6:266-270.

Costill DL, Thomas R, Robergs RA, Pascoe D, Lambert C, Barr S, Fink WJ. Adaptations to swimming training: influence of training volume. Med Sci Sports Exerc 1991;23:371-377.

Craig AB Jr, Pendergast DR. Relationships of stroke rate, distance per stroke, and velocity in competitive swimming. Med Sci Sports 1979; 11:278-283.

Craig AB Jr, Skehan PL, Pawelczyk JA, Boomer WL. Velocity, stroke rate, and distance per stroke during elite swimming competition. Med Sci Sports Exerc 1985;17:625-634.

Fulton SK, Pyne D, Hopkins W, Burkett B. Variability and progression in competitive performance of Paralympic swimmers. J Sports Sci 2009; 27:535-539.

Fulton SK, Pyne DB, Hopkins WG, Burkett B. Training characteristics of paralympic swimmers. J Strength Cond Res 2010;24:471-478.

Gehlsen GM, Karpuk J. Analysis of the NWAA swimming classification system. Adapt Phys Activ Q 1992;9:141-147.

Hellard P, Avalos M, Millet G, Lacoste L, Barale F, Chatard JC. Modeling the residual effects and threshold saturation of training: a case study of Olympic swimmers. J Strength Cond Res 2005;19:67-75.

Issurin VB. Generalized training effects induced by athletic preparation. A review. J Sports Med Phys Fitness 2009;49:333-345.
Kubukeli ZN, Noakes TD, Dennis SC. Training techniques to improve endurance exercise performances. Sports Med 2002;32:489-509.

Mujika I. The influence of training characteristics and tapering on the adaptation in highly trained individuals: a review. Int J Sports Med 1998;19:439-446.

Mujika I, Busso T, Lacoste L, Barale F, Geyssant A, Chatard JC. Modeled responses to training and taper in competitive swimmers. Med Sci Sports Exerc 1996a;28:251-258.

Mujika I, Chatard JC, Busso T, Geyssant A, Barale F, Lacoste L. Use of swim-training profiles and performance data to enhance training effectiveness. J Swim Res 1996b;11:23-29.

Mujika I, Padilla S. Scientific bases for precompetition tapering strategies. Med Sci Sports Exerc 2003;35:1182-1187.

Mujika I, Padilla S, Pyne D, Busso T. Physiological changes associated with the pre-event taper in athletes. Sports Med 2004;34:891-927.

Neary JP, Bhambhani YN, McKenzie DC. Effects of different stepwise reduction taper protocols on cycling performance. Can J Appl Physiol 2003a;28:576-587.

Neary JP, Martin TP, Quinney HA. Effects of taper on endurance cycling capacity and single muscle fiber properties. Med Sci Sports Exerc 2003b; 35:1875-1881.

Pelayo P, Sidney M, Moretto P, Wille F, Chollet D. Stroking parameters in top level swimmers with a disability. Med Sci Sports Exerc 1999;31: 1839-1843.

Pelayo P, Wille F, Sidney M, Berthoin S, Lavoie JM. Influence of age, sex, and anthropometric characteristics on velocity and stroking parameters in non skilled young swimmers. J Sports Med Phys Fit 1997;37: 187-193.

Schnitzler C, Seifert L, Chollet D. Variability of coordination parameters at 400-m front crawl swimming pace. J Sports Sci Med 2009;8:203-210.

Shepley B, MacDougall JD, Cipriano N, Sutton JR, Tarnopolsky MA, Coates G. Physiological effects of tapering in highly trained athletes. J Appl Physiol (1985) 1992;72:706-711.

Siff MC. Supertraining. 6th ed. Denve (Co): Supertraining Institute; 2004; 259-262.

Thomas L, Busso T. A theoretical study of taper characteristics to optimize performance. Med Sci Sports Exerc 2005;37:1615-1621.

Trappe S, Costill D, Thomas R. Effect of swim taper on whole muscle and single muscle fiber contractile properties. Med Sci Sports Exerc 2001; 33:48-56. 